\title{
EXISTENCE OF POSITIVE SOLUTIONS TO A COUPLED SYSTEM OF FRACTIONAL HYBRID DIFFERENTIAL EQUATIONS
}

\author{
Ghulam Hussain*, Rahmat Ali Khan and Muhammad Iqbal
}

Department of Mathematics, University of Malakand, Chakadara Dir (L), Khyber Pakhtunkhwa, Pakistan

*Corresponding Author Email: hussain4472@gmail.com

This is an open access article distributed under the Creative Commons Attribution License, which permits unrestricted use, distribution, and reproduction in any medium, provided the original work is properly cited.

\section{ARTICLE DETAILS}

Article History:

Received 18 November 2017 Accepted 12 December 2017 Available online 18 January 2018

\section{ABSTRACT}

In this article, we investigate existence and uniqueness of positive solution to coupled system of Caputo's boundary value problem for fractional order differential equations of the form

$$
\left\{\begin{array}{l}
D^{\alpha}[x(t)-f(t, x(t))]=g\left(t, y(t), I^{\alpha}(y(t))\right), t \in I=[0,1], \\
D^{\alpha}[y(t)-f(t, y(t))]=g\left(t, x(t), I^{\alpha}(x(t))\right), t \in I=[0,1], \\
D^{p} x(0)=\delta_{1} x\left(\eta_{1}\right), D^{p} x(1)=\delta_{2} x\left(\eta_{2}\right), 0<\eta_{1}, \eta_{2}<1, \\
D^{p} y(0)=\delta_{1} y\left(\eta_{1}\right), D^{p} y(1)=\delta_{2} y\left(\eta_{2}\right), 0<\eta_{1}, \eta_{2}<1, \quad 0<p<1,
\end{array}\right.
$$

where $\mathrm{D}^{\alpha}$ is the Caputo's fractional derivative of order $\alpha, 1<\alpha \leq 2, \mathrm{j}=[0, T], T>0$ and the functions $f: \mathrm{j} \times \mathrm{R} \times \mathrm{R} \rightarrow \mathrm{R}$, $f(0,0)=0$ and $g: j \times \mathrm{R} \times \mathrm{R} \rightarrow \mathrm{R}$ satisfy certain conditions. The proof of the existence theorem is based on a coupled fixed-point theorem of Krasnoselskii type, which extends a fixed-point theorem of Burton. Finally, our results are illustrated by providing a counter example.

\section{KEYWORDS}

Hybrid boundary value problem, Banach space, Coupled fixed point theorem, Caputo's fractional derivative.

\section{INTRODUCTION}

Fractional differential equations have extensive applications in real life problems. These applications can be found in various scientific and engineering disciplines such as physics, chemistry, biology, viscoelasticity, control theory, signal processing etc. [1-5]. Moreover, most of the authors also considered the fractional differential equations as an object of mathematical investigations, we refer the readers and the references therein for recent development of the theory [5-16]. Caputo's fractional derivatives play vital role in applied problems as it provides known physical interpretation for initial and boundary conditions. The development of various techniques in order to deduce the existence and other essential properties of the solution. There are still many open problems related the solvability of nonlinear system, apart from the fact that this is a field where advances are continuously taking place.

Some differential equation representing a certain dynamical system have no analytical solution, so the perturbation of such problem can be helpful. The perturbed differential equations are categorized into various types. An important type of these such perturbation is called a hybrid differential equation. Hybrid fixed point theory can be used to develop the existence theory for the hybrid equation [8]. Some researchers discussed the following first-order hybrid differential equation with linear perturbation of second type [13]:

$$
\left\{\begin{array}{l}
\frac{d}{d x}[x(t)-f(t, x(t))]=g(t, x(t)), t \in j, \\
x\left(t_{0}\right)=x_{0} \in \mathrm{R},
\end{array}\right.
$$

where $\left(j=t_{0}, t_{0}+\mathrm{a}\right)$, in $\mathrm{R}$ for some fixed $t_{0}, \mathrm{a} \in \mathrm{R}$ with $\mathrm{a}>0$, and $f, g$ $\in C(j \times \mathrm{R}, \mathrm{R})$. They proved the existence of the maximal and minimal solution for this equation.

A group of researchers, developed this problem as regard the following FHDEs involving the R-L differential operators of order $0<\mathrm{q}<1$, with linear perturbation of second type:

$$
\left\{\begin{array}{l}
D^{q}[x(t)-f(t, x(t))]=g(t, x(t)), t \in j \\
x\left(t_{0}\right)=x_{0} \in \mathrm{R}
\end{array}\right.
$$

where $f, g \in \mathrm{C}(j \times \mathrm{R}, \mathrm{R})$. Besides that, using mixed Lipschitz and Caratheodory condition allowed them to prove existence theorem for fractional hybrid differential equations.

The study of coupled system involving fractional differential equation is also important as such systems occurs in various problems of applied nature [17-20]. Lately, some group researchers, were discussed a twopoint boundary value problem for a coupled system fractional differential equation [21,22]. Other researchers, discussed the existence of solution of the fractional hybrid differential equations of order [23] $0<\alpha<1$ :

$$
\left\{\begin{array}{l}
D^{\alpha}[x(t)-f(t, x(t))]=g\left(t, y(t), I^{\alpha} y(t)\right), t \in j, \\
D^{\alpha}[y(t)-f(t, y(t))]=g\left(t, x(t), I^{\alpha} x(t)\right), t \in j, \\
x(0)=0, \quad y(0)=0 .
\end{array}\right.
$$

In line with the above works, our purpose in this paper is to prove the existence of solution to the following system of hybrid differential equation of order $1<\alpha<2$ : 


$$
\left\{\begin{array}{l}
D^{\alpha}[x(t)-f(t, x(t))]=g\left(t, y(t), I^{\alpha}(y(t))\right), t \in I=[0,1], \\
D^{\alpha}[y(t)-f(t, y(t))]=g\left(t, x(t), I^{\alpha}(x(t))\right), t \in I=[0,1], \\
D^{p} x(0)=\delta_{1} x\left(\eta_{1}\right), \quad D^{p} x(1)=\delta_{2} x\left(\eta_{2}\right), 0<\eta_{1}, \eta_{2}<1, \\
D^{p} y(0)=\delta_{1} y\left(\eta_{1}\right), \quad D^{p} y(1)=\delta_{2} y\left(\eta_{2}\right), 0<\eta_{1}, \eta_{2}<1, \quad 0<p<1 .
\end{array}\right.
$$

Where $1<\alpha \leq 2$. The proof is rooted in a coupled fixed-point theorem which is a generalization of a fixed-point theorem of Burton, in the Banach space [1]. By providing counter example, we deduce the results of our paper.

\section{PRELIMINARIES}

Let $C(j \times R, R)$ represent the class of continuous functions $f: j \times R \times \rightarrow R$ and let $C(j \times R \times R, R)$ denote the class of functions $g: j \times R \times R \rightarrow R$ such that

(i) For each $\mathrm{x}, \mathrm{y} \in \mathrm{R}$, the map $\mathrm{t} \rightarrow \mathrm{g}(\mathrm{t}, \mathrm{x}, \mathrm{y})$ is measurable.

(ii) For each $x \in R$, the map $x \rightarrow g(t, x, y)$ is continuous.

(iii) For each $\mathrm{y} \in \mathrm{R}$, the map $\mathrm{y} \rightarrow \mathrm{g}(\mathrm{t}, \mathrm{x}, \mathrm{y})$ is continuous.

The class $C(j \times R \times R, R)$ is called the Caratheodory class of functions on $j$ $\times \mathrm{R} \times \mathrm{R}$, which are Lebesgue integrable when bounded by Lebesgue integrable function on $\mathrm{j}$. In this section, we recall some basic definitions, lemmas and notations.

Definition 2.1. The fractional integral of order $\mathrm{q} \in \mathrm{R}+$ of the function $\mathrm{h} \in$ $\mathrm{L} 1([\mathrm{a}, \mathrm{b}], \mathrm{R})$ is defined as

$$
I_{a+}^{q} h(t)=\frac{1}{\Gamma(q)} \int_{a}^{t}(t-s)^{q-1} h(s) d s,
$$

provided that integral on the right is pointwise defined on $(0, \infty)$.

Definition 2.2. The Caputo fractional order derivative of a function $h$ on the interval $[a, b]$ is defined by

$$
{ }^{c} D_{a+}^{q} h(t)=\frac{1}{\Gamma(n-q)} \int_{a}^{t}(t-s)^{n-q-1} h^{(n)}(s)^{2} d s,
$$

where $\mathrm{n}=[\mathrm{q}]+1$.

Lemma 2.3. Let $0<\alpha<1$ and $\mathrm{f} \in \in^{1}(0,1)$. Then

$\left(K_{1}\right)$. The equality $D^{\alpha} I^{\alpha} f(t)=f(t)$;

(K2). The equality $I^{\alpha} D^{\alpha} f(t)=f(t)-\frac{\left[D^{\alpha-1} f(t)\right]_{l=0}}{\Gamma(\alpha)} t^{\alpha-1}$, hold almost everywhere on J.

The following fixed-point theorem in Banach spaces due to Burton is necessary for our study though out in this paper.

Theorem 2.4 Let $S$ be a non-empty, closed, convex and bounded sub set of a Banach space $\mathrm{X}$ and $\widetilde{S}=S \times S$. Suppose that $\mathrm{A}: \mathrm{X} \rightarrow \mathrm{X}$ and $\mathrm{B}: \mathrm{S} \rightarrow \mathrm{X}$ be two operators such that (C1) there exists $\varphi_{\mathrm{A}} \in \phi$ such that for all $\mathrm{x}, \mathrm{y}$ $\in X$, we have

$$
\|A x-A y\| \leq \sigma \varphi_{A}(\|x-y\|),
$$

for some constant $\sigma>0$,

(C2) B is completely continuous,

(C3) $\mathrm{x}=\mathrm{Ax}+\mathrm{By} \Rightarrow \mathrm{x} \in \mathrm{S}$ for all $\mathrm{y} \in \mathrm{S}$.

Then the operator equation $\mathrm{Ax}+\mathrm{By}=\mathrm{x}$ has a solution in $\mathrm{S} \sim$, whenever $\sigma<1$.

Definition 2.5 An element $(x, y) \in X \times X$ is called a coupled fixed point of a mapping $\mathrm{T}: \mathrm{X} \times \mathrm{X} \rightarrow \mathrm{X}$ if $\mathrm{T}(\mathrm{x}, \mathrm{y})=\mathrm{x}$ and $\mathrm{T}(\mathrm{y}, \mathrm{x})=\mathrm{y}$.

\section{MAIN RESULT}

In this section, let $\mathrm{X}=\mathrm{C}(\mathrm{j}, \mathrm{R})$ equipped with the supremum norm defined by $\|x\|=\sup \{x(t):|x(t)|\}$. Clearly $(X,\|x\|$ is a Banach space. In the

following lemma we introduce a certain Banach space which is used in our results.

Lemma 3.1. Let $\widetilde{X}=X \times X$. Define
Then $\widetilde{X}$ is Banach space with respect to the above norm. Now, we prove a coupled fixed-point theorem which is generalization of Theorem 2.4, we study the existence of solution for the FHDEs system (1.1) under the following general assumptions.

(H0) The function $x \rightarrow x-f(t, x)$ is increasing in R for all $t \in J$

(H1) There exists a constant $M \geq L>0$ such that

$$
\begin{aligned}
& \quad|f(t, x)-f(t, y \quad)| \leq \frac{L\|x-y\|}{2(M+\|x-y\|)} \text {, for all } t \in j \text { and } x, y \in \mathrm{R} \\
& \text { (H2) Setting } \quad F_{0}=\max |f(t 0)|
\end{aligned}
$$

(H3) There exists a continuous function $h \in C(J, R)$ such that $g(t, x(t), I \alpha$ $x(t)) \leq h(t), t \in j$ and $x, y \in R$.

Lemma 3.2 Assume that hypothesis ( $H 0$ ) holds, $y \in C(j, R), 0<p<1,1<\alpha \leq$ 2 and $f \in C(j \times R, R)$ with $f(0,0)=0$. Then the unique solution of the boundary value problem

$$
\left\{\begin{array}{l}
D^{\alpha}[x(t)-f(t, x(t))]=h(t), \\
D^{p} x(0)=\delta_{1} x\left(\eta_{1}\right), D^{p} x(1)=\delta_{2} x\left(\eta_{2}\right), 0<\eta_{1}, \eta_{2}<1,
\end{array}\right.
$$

and $\delta_{1}, \delta_{2}$ are non-zero number has a solution such that

$$
\begin{aligned}
x(t) & =f(t, x(t))-f\left(\eta_{1}, x\left(\eta_{1}\right)\right)-\frac{\left(\eta_{1}-t\right)}{\delta_{2}\left[\eta_{2}-\eta_{1}-\frac{1}{\delta_{1}(2-p)}\right]}\left[D^{p} f(1, x(1))\right. \\
& \left.-\delta_{2}\left\{f\left(\eta_{2}, x\left(\eta_{2}\right)\right)-f\left(\eta_{1}, x\left(\eta_{1}\right)\right)\right\}\right]+I^{\alpha} g\left(t, y(t), I^{\alpha} y(t)\right)-I^{\alpha} g\left(\eta_{1}, y\left(\eta_{1}\right), I^{\alpha} y\left(\eta_{1}\right)\right) \\
& -\frac{\left(\eta_{1}-t\right)}{\delta_{2}\left[\eta_{2}-\eta_{1}-\frac{1}{\delta_{2}(l-p)}\right]}\left[I^{\alpha-p} g\left(1, y(1), I^{\alpha} y(1)\right)-\delta_{2}\left\{I^{\alpha} g\left(\eta_{2}, y\left(\eta_{2}\right), I^{\alpha} y\left(\eta_{2}\right)\right)\right.\right. \\
& \left.-I^{\alpha} g\left(\eta_{1}, y\left(\eta_{1}\right), I^{\alpha} y\left(\eta_{1}\right)\right)\right] .
\end{aligned}
$$

Proof. Set $X=C(j, \mathrm{R})$ and a sub set $S$ of $X$ defined by $S=\{x \in X:\|x\| \leq N\}$, where $N \geq 2\left(L+F_{0}+\frac{\|h\|}{\Gamma(\alpha+1)}\right)$. Clearly $\mathrm{S}$ is a closed, convex and bounded sub set of a Banach space X. Now we take the system (1.1) that has a solution $x(t)$ if and only if $x(t)$ satisfied the following system of integral equations. We assume for the solution of given problem

$$
D^{\alpha}[x(t)-f(t, x(t))]=h(t) .
$$

Now apply ${ }^{\alpha}{ }^{\alpha}$ to both sides we get

$$
x(t)=f(t, x(t))+I^{\alpha} h(t)+c_{0}+c_{1} t
$$

for some $c_{0}, c_{1} \in \mathrm{R}$. The boundary conditions $D^{\alpha} x(0)=\delta_{1} x\left(\eta_{1}\right)$ and $D^{\alpha} x(1)=\delta 2 x(\eta 2)$ implies

$$
\begin{aligned}
c_{1} & =\frac{1}{\delta_{1}\left[\eta_{2}-\eta_{1}-\frac{1}{\delta_{2} \Gamma(2-p)}\right]} D^{p} f(1, x(1))+ \\
- & \delta_{2}\left\{f \left(\eta_{2}, x\left(\eta_{2}\right)-f\left(\eta_{1}, x\left(\eta_{1}\right)+I^{\alpha} h\left(\eta_{2}\right)-I^{\alpha} h\left(\eta_{1}\right)\right\}\right.\right. \\
c_{0}= & f\left(\eta_{1}, x\left(\eta_{1}\right)-I^{\alpha} h\left(\eta_{1}\right)\right. \\
& -\frac{\eta_{1}}{\delta_{2}\left[\eta_{2}-\eta_{1}-\frac{1}{\delta_{2} \Gamma(2-p)}\right]} D^{p} f(1, x(1))+I^{\alpha-p} h(1) \\
& -\delta_{2}\left\{f \left(\eta_{2}, x\left(\eta_{2}\right)-f\left(\eta_{1}, x\left(\eta_{1}\right)+I^{\alpha} h\left(\eta_{2}\right)-I^{\alpha} h\left(\eta_{1}\right)\right\}\right.\right.
\end{aligned}
$$

It follows that

$$
\begin{aligned}
x(t) & =f(t, x(t))-f\left(\eta_{1}, x\left(\eta_{1}\right)\right)-\frac{\left(\eta_{1}-t\right)}{\delta_{2}\left[\eta_{2}-\eta_{1}-\frac{1}{\delta_{2} \Gamma(2-p)}\right]}\left[D^{p} f(1, x(1))\right. \\
& \left.-\delta_{2}\left\{f\left(\eta_{2}, x\left(\eta_{2}\right)\right)-f\left(\eta_{1}, x\left(\eta_{1}\right)\right)\right\}\right]+I^{\alpha} g\left(t, y(t), I^{\alpha} y(t)\right)-I^{\alpha} g\left(\eta_{1}, y\left(\eta_{1}\right), I^{\alpha} y\left(\eta_{1}\right)\right) \\
& -\frac{\left(\eta_{1}-t\right)}{\delta_{2}\left[\eta_{2}-\eta_{1}-\frac{1}{\delta_{2} \Gamma(2-p)}\right]}\left[I^{\alpha-p} g\left(1, y(1), I^{\alpha} y(1)\right)-\delta_{2}\left\{I^{\alpha} g\left(\eta_{2}, y\left(\eta_{2}\right), I^{\alpha} y\left(\eta_{2}\right)\right)\right.\right. \\
& \left.-I^{\alpha} g\left(\eta_{1}, y\left(\eta_{1}\right), I^{\alpha} y\left(\eta_{1}\right)\right)\right] .
\end{aligned}
$$

$$
\|(x, y)\|=\|x\|+\|y\| .
$$


Similarly, we determined

$$
\begin{aligned}
y(t) & =f(t, y(t))-f\left(\eta_{1}, y\left(\eta_{1}\right)\right)-\frac{\left(\eta_{1}-t\right)}{\delta_{2}\left[\eta_{2}-\eta_{1}-\frac{1}{\delta_{\Gamma} \Gamma(2-p)}\right]}\left[D^{p} f(1, y(1))-\delta_{2}\left\{f\left(\eta_{2}, y\left(\eta_{2}\right)\right)\right.\right. \\
& \left.\left.-f\left(\eta_{1}, y\left(\eta_{1}\right)\right)\right\}\right]+I^{\alpha} g\left(t, x(t), I^{\alpha} x(t)\right)-I^{\alpha} g\left(\eta_{1}, x\left(\eta_{1}\right), I^{\alpha} x\left(\eta_{1}\right)\right) \\
& -\frac{\left(\eta_{1}-t\right)}{\delta_{2}\left[\eta_{2}-\eta_{1}-\frac{1}{\delta_{2}(2-2)}\right]}\left[I^{\alpha-p} g\left(1, x(1), I^{\alpha} x(1)\right)-\delta_{2}\left\{I^{\alpha} g\left(\eta_{2}, x\left(\eta_{2}\right), I^{\alpha} x\left(\eta_{2}\right)\right)\right.\right. \\
& \left.-I^{\alpha} g\left(\eta_{1}, x\left(\eta_{1}\right), I^{\alpha} x\left(\eta_{1}\right)\right)\right] .
\end{aligned}
$$

Now we need to prove the existence theorem for the FHDEs of the system (1.1). Define two operators A $: X \rightarrow X$ and B : $\rightarrow X$

$$
\begin{aligned}
A x(t) & =f(t, x(t))-f\left(\eta_{1}, x\left(\eta_{1}\right)\right)-\frac{\left(\eta_{1}-t\right)}{\delta_{2}\left[\eta_{2}-\eta_{1}-\frac{1}{\delta_{2} \Gamma(2-p)}\right]}\left[D^{p} f(1, x(1))\right. \\
& \left.-\delta_{2}\left\{f\left(\eta_{2}, x\left(\eta_{2}\right)\right)-f\left(\eta_{1}, x\left(\eta_{1}\right)\right)\right\}\right]
\end{aligned}
$$

and

$$
\begin{aligned}
B x(t) & =I^{\alpha} g\left(t, x(t), I^{\alpha} x(t)\right)-I^{\alpha} g\left(\eta_{1}, x\left(\eta_{1}\right), I^{\alpha} x\left(\eta_{1}\right)\right) \\
& -\frac{\left(\eta_{1}-t\right)}{\delta_{2}\left[\eta_{2}-\eta_{1}-\frac{1}{\delta_{2} \Gamma(2-p)}\right]}\left[I^{\alpha-p} g\left(1, x(1), I^{\alpha} x(1)\right)\right. \\
& -\delta_{2}\left\{I^{\alpha} g\left(\eta_{2}, x\left(\eta_{2}\right), I^{\alpha} x\left(\eta_{2}\right)\right)-I^{\alpha} g\left(\eta_{1}, x\left(\eta_{1}\right), I^{\alpha} x\left(\eta_{1}\right)\right)\right] .
\end{aligned}
$$

so, the system $x(t)$ and $y(t)$ are transformed into the system of operator equations as

$$
\left\{\begin{aligned}
x(t) & =A x(t)+B y(t), \\
& =A y(t)+B x(t) .
\end{aligned}\right.
$$

We shall show that the operators A and B satisfy all the conditions of Theorem (2.4). Let $\mathrm{x}, \mathrm{y} \in \mathrm{X}$, by hypothesis (H1), we have

$|A x(t)-A y(t)|=\mid f(t, x(t))-f(t, y(t))$

$$
\begin{aligned}
& -\left\{f\left(\eta_{1}, x\left(\eta_{1}\right)\right)-f\left(\eta_{1}, y\left(\eta_{1}\right)\right)\right\}-\frac{\left(\eta_{1}-t\right)}{\delta_{2}\left[\eta_{2}-\eta_{1}-\frac{1}{d_{1},(2-p)}\right]}\left[D^{p} f(1, x(1))-D^{p} f(1, y(1))\right. \\
& \left.-\delta_{2}\left\{f\left(\eta_{2}, x\left(\eta_{2}\right)\right)-f\left(\eta_{2}, y\left(\eta_{2}\right)\right)-f\left(\eta_{1}, x\left(\eta_{1}\right)\right)+f\left(\eta_{1}, x\left(\eta_{1}\right)\right)\right\}\right] \mid \\
\leq & |f(t, x(t))-f(t, y(t))|+\left|f\left(\eta_{1}, x\left(\eta_{1}\right)\right)-f\left(\eta_{1}, y\left(\eta_{1}\right)\right)\right|+\mid \frac{\left(\eta_{1}-t\right)}{d e l t a_{2}\left[\eta_{2}-\eta_{1}-\frac{1}{\delta_{1},(2-p)}\right]} \\
& \mid\left[\left|D^{p} f(1, x(1))-D^{p} f(1, y(1))\right|+\left|\delta_{2}\right|\left\{\left|f\left(\eta_{2}, x\left(\eta_{2}\right)\right)-f\left(\eta_{2}, y\left(\eta_{2}\right)\right)\right|\right.\right. \\
& \left.\left.+\left|f\left(\eta_{1}, x\left(\eta_{1}\right)\right)+f\left(\eta_{1}, x\left(\eta_{1}\right)\right)\right|\right\}\right] \\
\leq & \frac{L(|x(t)-y(t)|}{4(M+|x(t)-y(t)|)}+\frac{1}{\left|\delta_{2}\left[\eta_{2}-\eta_{1}-\frac{1}{\delta_{1},(2-p)}\right]\right|} \\
& {\left[\left|D^{p} f(1, x(1))-D^{p} f(1, y(1))\right|+\left|\delta_{2}\right| \frac{L\left(\left|x\left(\eta_{2}\right)-y\left(\eta_{2}\right)\right|\right.}{4\left(M+\left|x\left(\eta_{2}\right)-y\left(\eta_{2}\right)\right|\right)}\right] }
\end{aligned}
$$

taking supremum over t we obtain

$$
\begin{aligned}
\|A x-A y\| & \leq \frac{L(\|x-y\|)}{4(M+\|x-y\|)}+\frac{1}{\left|\delta_{2}\left[\eta_{2}-\eta_{1}-\frac{1}{\delta_{2} \Gamma(2-p)}\right]\right|} \mid\left[\frac{\delta_{2} \mid L(\|x-y\|)}{4(M+\|x-y\|)}\right. \\
& \left.+\frac{\left|\delta_{2}\right| L(\|x-y\|)}{4(M+\|x-y\|)}\right] \\
\leq & \frac{L(\|x-y\|)}{4(M+\|x-y\|)}\left[1+\frac{2}{\left|\left[\eta_{2}-\eta_{1}-\frac{1}{\delta_{2}(2-p)}\right]\right|}\right] \\
\leq & \frac{L(\|x-y\|)}{2(M+\|x-y\|)}
\end{aligned}
$$

This shows that $A$ is a contraction on $X$ with function $\frac{1}{2} \phi$ where $\phi$ is given by $\phi(r)=\frac{L r}{M+r}$. Let $\{x\}$ be a sequence in $S$ converging to a point $x \in S$.

Then

$$
\begin{aligned}
B x_{n}(t) & =I^{\alpha} g\left(t, x_{n}(t), I^{\alpha} x_{n}(t)\right)-I^{\alpha} g\left(\eta_{1}, x_{n}\left(\eta_{1}\right), I^{\alpha} x_{n}\left(\eta_{1}\right)\right) \\
& -\frac{\left(\eta_{1}-t\right)}{\delta_{2}\left[\eta_{2}-\eta_{1}-\frac{1}{\delta_{2} \Gamma(2-p)}\right]} I^{\alpha-p} g\left(1, x_{n}(1), I^{\alpha} x_{n}(1)\right) \\
& -\delta_{2}\left\{I^{\alpha} g\left(\eta_{2}, x_{n}\left(\eta_{2}\right), I^{\alpha} x_{n}\left(\eta_{2}\right)\right)-I^{\alpha} g\left(\eta_{1}, x_{n}\left(\eta_{1}\right), I^{\alpha} x_{n}\left(\eta_{1}\right)\right)\right\} \\
& =I^{\alpha} g\left(t, x(t), I^{\alpha} x(t)\right)-I^{\alpha} g\left(\eta_{1}, x\left(\eta_{1}\right), I^{\alpha} x\left(\eta_{1}\right)\right) \\
& -\frac{\left(\eta_{1}-t\right)}{\delta_{2}\left[\eta_{2}-\eta_{1}-\frac{1}{\delta_{2} \Gamma(2-p)}\right]} I^{\alpha-p} g\left(1, x(1), I^{\alpha} x(1)\right) \\
& -\delta_{2}\left\{I^{\alpha} g\left(\eta_{2}, x\left(\eta_{2}\right), I^{\alpha} x\left(\eta_{2}\right)\right)-I^{\alpha} g\left(\eta_{1}, x\left(\eta_{1}\right), I^{\alpha} x\left(\eta_{1}\right)\right)\right\} \\
& =B x(t)
\end{aligned}
$$

for all $t \in J$, where the second equality holds by Lebesgue dominated convergence theorem. So $B$ is continuous function on $S$. Let $x \in S$, by assumption (H2), for $t \in J$, we have

$$
\begin{aligned}
& |B x(t)|=\mid I^{\alpha} g\left(t, x(t), I^{\alpha} x(t)\right)-I^{\alpha} g\left(\eta_{1}, x\left(\eta_{1}\right), I^{\alpha} x\left(\eta_{1}\right)\right) \\
& -\frac{\left(\eta_{1}-t\right)}{\delta_{2}\left[\eta_{2}-\eta_{1}-\frac{1}{\delta_{2} \Gamma(2-p)}\right]} I^{\alpha-p} g\left(1, x(1), I^{\alpha} x(1)\right) \\
& -\delta_{2}\left\{I^{\alpha} g\left(\eta_{2}, x\left(\eta_{2}\right), I^{\alpha} x\left(\eta_{2}\right)\right)-I^{\alpha} g\left(\eta_{1}, x\left(\eta_{1}\right), I^{\alpha} x\left(\eta_{1}\right)\right)\right\} \mid \\
& \leq\left[\left|I^{\alpha} g\left(t, x(t), I^{\alpha} x(t)\right)\right|+\left|I^{\alpha} g\left(\eta_{1}, x\left(\eta_{1}\right), I^{\alpha} x\left(\eta_{1}\right)\right)\right|\right] \\
& +\mid \frac{\left(\eta_{1}-t\right)}{\delta_{2}\left[\eta_{2}-\eta_{1}-\frac{1}{\delta_{2} \Gamma(2-p)}\right]}\left[\left|I^{\alpha-p} g\left(1, x(1), I^{\alpha} x(1)\right)\right|\right. \\
& +\left|\delta_{2}\right|\left\{\left|I^{\alpha} g\left(\eta_{2}, x\left(\eta_{2}\right), I^{\alpha} x\left(\eta_{2}\right)\right)-I^{\alpha} g\left(\eta_{1}, x\left(\eta_{1}\right), I^{\alpha} x\left(\eta_{1}\right)\right)\right|\right\} \text {. }
\end{aligned}
$$

Now

$$
\begin{aligned}
\mid I^{\alpha} g(t, x(t), & \left.I^{\alpha} x(t)\right)\left|=\frac{1}{\Gamma \alpha}\right| \int_{0}^{t}(t-s)^{\alpha-1} g\left(s, x(s), I^{\alpha} x(s)\right) d s \mid \\
& \leq \frac{1}{\Gamma \alpha} \int_{0}^{t}(t-s)^{\alpha-1}\left|g\left(s, x(s), I^{\alpha} x(s)\right)\right| d s \\
& \leq \frac{1}{\Gamma \alpha} \int_{0}^{t}(t-s)^{\alpha-1}\|h\| d s \\
& \leq \frac{\|h\|}{\Gamma(\alpha+1)} t^{\alpha} .
\end{aligned}
$$

Similarly, $\left|I^{\alpha} g\left(\eta_{1}, x\left(\eta_{1}\right), I^{\alpha} x\left(\eta_{1}\right)\right)\right| \leq \frac{\|h\|}{\Gamma(\alpha+1)} \eta_{1}^{\alpha}$ and

$\left|I^{\alpha} g\left(\eta_{2}, x\left(\eta_{2}\right), I^{\alpha} x\left(\eta_{2}\right)\right)\right| \leq \frac{\|h\|}{\Gamma(\alpha+1)} \eta_{2}^{\alpha},\left|I^{\alpha-p} g\left(1, x(1), I^{\alpha} x(1)\right)\right| \leq \frac{\|h\|}{\Gamma(\alpha-p+1)}$ Therefore (3.2) implies that

$$
\|B x(t)\| \leq\|h\|\left[\frac{4}{\Gamma(\alpha+1)}+\left|\frac{1}{\delta_{2}\left[\eta_{2}-\eta_{1}-\frac{1}{\delta_{\Gamma} \Gamma(2-p)}\right]}\right| \frac{1}{\Gamma(\alpha-p+1)}\right]
$$

for all $\mathrm{x} \in \mathrm{S}$, so $\mathrm{B}$ is uniformly bounded on $\mathrm{S}$.

Now, let $\mathrm{t}_{1}, \mathrm{t}_{2} \in \mathrm{J}$. For any $\mathrm{x} \in \mathrm{S}$, we have

$$
\begin{aligned}
\left|B x\left(t_{1}\right)-B x\left(t_{2}\right)\right| & =\mid I^{\alpha} g\left(t_{1}, x\left(t_{1}\right), I^{\alpha} x\left(t_{1}\right)\right)-I^{\alpha} g\left(t_{2}, x\left(t_{2}\right), I^{\alpha} x\left(t_{2}\right)\right) \\
& +\frac{\left(t_{1}-t_{2}\right)}{\delta_{2}\left[\eta_{2}-\eta_{1}-\frac{1}{\delta_{2} \Gamma(2-p)}\right]}\left[I^{\alpha-p} g\left(1, x(1), I^{\alpha} x(1)\right)\right. \\
& -\delta_{2}\left\{I^{\alpha} g\left(\eta_{2}, x\left(\eta_{2}\right), I^{\alpha} x\left(\eta_{2}\right)\right)-I^{\alpha} g\left(\eta_{1}, x\left(\eta_{1}\right), I^{\alpha} x\left(\eta_{1}\right)\right)\right] \mid
\end{aligned}
$$

Now

$\left|B x\left(t_{1}\right)-B x\left(t_{2}\right)\right| \leq\left|I^{\alpha} g\left(t_{1}, x\left(t_{1}\right), I^{\alpha} x\left(t_{1}\right)\right)-I^{\alpha} g\left(t_{2}, x\left(t_{2}\right), I^{\alpha} x\left(t_{2}\right)\right)\right|$

$$
\begin{aligned}
& +\left|\frac{t_{1}-t_{2}}{\delta_{2}\left[\eta_{2}-\eta_{1}-\frac{1}{\delta_{2} \Gamma(2-p)}\right]}\right| I^{\alpha-p} g\left(1, x(1), I^{\alpha} x(1)\right) \mid \\
& +\left|\delta_{2}\right|\left\{I^{\alpha} g\left(\eta_{2}, x\left(\eta_{2}\right), I^{\alpha} x\left(\eta_{2}\right)\right)-I^{\alpha} g\left(\eta_{1}, x\left(\eta_{1}\right), I^{\alpha} x\left(\eta_{1}\right)\right) \mid\right. \\
& \leq \frac{1}{\Gamma \alpha} \int_{0}^{t}\left(t_{1}-s\right)^{\alpha-1} g\left(s, x(s), I^{\alpha} x(s)\right) d s-\int_{0}^{t}\left(t_{2}-s\right)^{\alpha-1} g\left(s, x(s), I^{\alpha} x(s)\right) d s \mid \\
& +\frac{t_{1}-t_{2}}{\delta_{2}\left[\eta_{2}-\eta_{1}-\frac{1}{\delta_{2}(2-p)}\right]} \frac{1}{\Gamma(\alpha-p)} \int_{0}^{1}(1-s)^{\alpha-p-1} g\left(s, x(s), I^{\alpha} x(s)\right) d s \\
& +\delta_{2} \frac{1}{\Gamma \alpha} \int_{0}^{\eta_{1}}\left(\eta_{2}-s\right)^{\alpha-1} g\left(\eta_{2}, x\left(\eta_{2}\right), I^{\alpha} x\left(\eta_{2}\right)\right) d s \\
& -\frac{1}{\Gamma \alpha} \int_{0}^{\eta_{1}}\left(\eta_{1}-s\right)^{\alpha-1} g\left(\eta_{1}, x\left(\eta_{1}\right), I^{\alpha} x\left(\eta_{1}\right)\right) d s \\
& \leq \frac{\|h\|}{(\alpha+1)}\left(t_{1}^{\alpha}-t_{2}^{\alpha}\right)+\frac{\left(t_{1}-t_{2}\right)}{\delta_{2}\left[\eta_{2}-\eta_{1}-\frac{1}{\left.\delta_{2}(2-2)\right]}\right]}\left|\frac{\|h\|}{(\alpha-p+1)}+\delta_{2} \frac{\|h\|}{(\alpha+1)}\left(\eta_{2}^{\alpha}-\eta_{1}^{\alpha}\right)\right| \\
& <\|h\| \frac{\alpha \delta}{(\alpha+1)}+\frac{\delta}{\delta_{2}\left[\eta_{2}-\eta_{1}-\frac{1}{\delta_{1}(2-p)}\right]}\left(\frac{1}{(\Gamma \alpha-p+1)}+\frac{\delta_{2}}{(\alpha+1)}\left(\eta_{2}^{\alpha}-\eta_{1}^{\alpha}\right)\right)=\delta \Delta \text {, }
\end{aligned}
$$

where

$$
\Delta=\|h\| \frac{1}{\Gamma \alpha}+\frac{1}{\delta_{2}\left[\eta_{2}-\eta_{1}-\frac{1}{\delta_{2} \Gamma(2-p)}\right]}\left(\frac{1}{(\Gamma \alpha-p+1)}+\frac{\delta_{2}}{(\alpha+1)}\left(\eta_{2}^{\alpha}-\eta_{1}^{\alpha}\right)\right)
$$

Therefore, $\left|B x\left(t_{l}\right)-B x\left(t_{2}\right)\right|<\delta \Delta=\varepsilon$ where

$\delta=\frac{\varepsilon}{\Delta}$. This implies, $\left.\mid\right) \mid<\varepsilon^{B x}\left(t_{1}\right)-B x\left(t_{2}\right)$ whenever $\left|t_{1}-t_{2}\right|<\delta$,

Thus $\mathrm{B}(\mathrm{S})$ is equi-continuous. Hence $\mathrm{B}$ is completely continuous on $\mathrm{S}$. Let $\mathrm{x}$ $\in X$ and $y \in S$ such that $x=A x+B y$. Now 


$$
|x(t)| \leq|A x(t)|+|B y(t)|
$$

$|x(t)| \leq|f(t, x(t))|+\left|f\left(\eta_{1}, x\left(\eta_{1}\right)\right)\right|+\left|I^{\alpha} g\left(t, y(t), I^{\alpha} y(t)\right)\right|+\left|I^{\alpha} g\left(\eta_{1}, y\left(\eta_{1}\right), I^{\alpha} y\left(\eta_{1}\right)\right)\right|$

Also

$$
\begin{aligned}
|x(t)| & \leq|f(t, x(t))-f(t, 0)|+|f(t, 0)|+\left|f\left(\eta_{1}, x\left(\eta_{1}\right)\right)-f(t, 0)\right|+|f(t, 0)| \\
& +\left|I^{\alpha} g\left(t, y(t), I^{\alpha} y(t)\right)\right|+\left|I^{\alpha} g\left(\eta_{1}, y\left(\eta_{1}\right), I^{\alpha} y\left(\eta_{1}\right)\right)\right| \\
& \leq \frac{L\left(\left|x\left(\eta_{1}\right)\right|\right.}{2\left(M+\left|x\left(\eta_{1}\right)\right|\right)}+F_{o}+\frac{L\left(\left|x\left(\eta_{1}\right)\right|\right.}{2\left(M+\left|x\left(\eta_{1}\right)\right|\right)}+F_{o}+\frac{\|h\|}{\Gamma(\alpha+1)} t^{\alpha}+\frac{\|h\|}{\Gamma(\alpha+1)} \eta_{1}^{\alpha} \\
& \leq L+F_{0}+L+F_{0}+\frac{\|h\|}{\Gamma(\alpha+1)}+\frac{\|h\|}{\Gamma(\alpha+1)} \\
& \leq 2\left(L+F_{o}+\frac{\|h\|}{\Gamma(\alpha+1)}\right) \leq N
\end{aligned}
$$

Hence $\|x\| \leq 2\left(L+F_{o}+\frac{\|h\|}{\Gamma(\alpha+1)}\right) \leq N$. Thus $x \in S$.

As all the conditions are satisfied, hence the given system has a solution.

\section{EXAMPLE}

Example 4.1. Consider the following coupled system of HFDEs

$$
\left\{\begin{array}{l}
{ }^{c} D^{\frac{3}{2}}\left[x(t)-\left(\frac{t^{2}}{80}+\frac{t|x(t)|}{20+|x(t)|}\right)\right]=\frac{t^{2}}{20}+y(t)+\cos \left|I^{\frac{3}{2}} y(t)\right|, \quad t \in[0,1], \\
{ }^{c} D^{\frac{3}{2}}\left[y(t)-\left(\frac{t^{2}}{80}+\frac{t|y(t)|}{20+|y(t)|}\right)\right]=\frac{t^{2}}{20}+x(t)+\cos \left|I^{\frac{3}{2}} x(t)\right|, \quad t \in[0,1], \\
{ }^{c} D^{\frac{1}{2}} x(0)=\frac{1}{2} x\left(\frac{1}{2}\right), D^{\frac{1}{2}} x(1)=\frac{1}{3} x\left(\frac{1}{3}\right), \\
{ }^{c} D^{\frac{1}{2}} y(0)=\frac{1}{2} y\left(\frac{1}{2}\right), D^{\frac{1}{2}} y(1)=\frac{1}{3} y\left(\frac{1}{3}\right) .
\end{array}\right.
$$

The solution of the BVP is given by

$$
\begin{gathered}
f(t, x)=\frac{t|x(t)|}{20+|x(t)|} \\
|f(t, x)-f(t, y)| \leq \frac{\|x-y\|}{20+\|x-y\|} \\
L=1, F_{0}=\sup |f(t, 0)|=\frac{1}{80},\|h\|=\frac{1}{60} .
\end{gathered}
$$

Therefore, $2\left(L+F_{0^{+}} \frac{\|h\|}{\Gamma \alpha+}\right)=2\left(1+\frac{1}{80}+\frac{1}{45 \pi}\right)=2.05<3=N$

So $N=3$. Hence, by the above theorem we conclude that the problem has a solution in $S=\{x \in X:\|x\| \leq 3\}$.

\section{REFERENCES}

[1] Hilfer, R. 2000. Applications of Fractional Calculus in Physics, World Scientific, Singapore.

[2] Kilbas, A. A., Marichev, O.I., Samko, S.G. 1993. Fractional Integrals and Derivatives (Theory and Applications), Gordon and Breach, Switzerland.

[3] Kilbas, A.A., Srivastava, H.M., Trujillo, J.J. 2006. Theory and Applications of Fractional Differential Equations, North-Holland mathematics Studies, 204, Elsevier, Amsterdam.

[4] Miller, K.S., Ross, B. 1993. An Introduction to the Fractional Calculus and Fractional Differential Equations, Wiley, New York.

[5] Podlubny, I. 1999. Fractional Differential Equations, Mathematics in Science and Engineering, Academic Press, New York.

[6] Belarbi, A., Benchohra, M., Ouahab, A. 2006. Existence results for functional differential equations of fractional order. Applicable Analysis, 85 (2), 1459-1470.

[7] Agarwal, R.P., Benchohra, M., Hamani, S. 2008. Boundary value problems for differential inclusions with fractional order. Advanced Studies in Contemporary Mathematics, 12 (2), 181-196.
[8] Benchohra, M., Graef, J.R., Hamani, S. 2008. Existence results for boundary value problems with nonlinear fractional differential equations. Applicable Analysis, 87 (2), 851-863.

[9] Benchohra, M., Hamani, S., Ntouyas, S.K. 2008. Boundary value problems for differential equations with fractional order. Surveys in Mathematics and its Applications, 3 (2), 1-12.

[10] Benchohra, M., Henderson, J., Ntouyas, S.K., Ouahab, A. 2008. Existence results for fractional order functional differential equations with infinite delay. Journal of Mathematical Analysis and Applications, 332 (2), 1340-1350.

[11] Agarwal, R.P., Benchohra, M., Hamani, S. 2009. Boundary value problems for fractional differential equations. Georgian Mathematical Journal, 12 (2), 401-411.

[12] Ahmad, B. Nieto, J.J. 2009. Existence results for nonlinear boundary value problems of fractional integrodifferential equations with integral boundary conditions. Boundary Value Problem Art., 708576, pp. 11.

[13] Ahmad, B., Otero-Espinar, V. 2009. Existence of solutions for fractional differential inclusions with anti-periodic boundary conditions. Boundary Value Problem Art., 625347, pp. 11.

[14] Lakshmikantham, V., Leela, S., Vasundhara, J. 2009. Theory of Fractional Dynamic Systems, Cambridge Academic Publishers, Cambridge, UK.

[15] Agarwal, R.P., Benchohra, M., Hamani, S. 2010. A Survey on Existence Results for Boundary Value Problems of Nonlinear Fractional Differential Equations and Inclusions. Acta Applicandae Mathematicae, 109 (1), 973-1033.

[16] Su, X., Zhang, S. 2011. Unbounded solutions to a boundary value problem of fractional order on the half-line. Computers \& Mathematics with Applications, 61 (2), 1079-1087.

[17] El-Shahed, M., Shammakh, W.M. 2011. Existence of positive solutions for m-point boundary value problem for nonlinear fractional differential equation. Abstract and Applied Analysis, Article ID 986575, pp. 20.

[18] Cui, Z., Yu, P., Mao, Z. 2012. Existence of solutions for nonlocal boundary value problems of nonlinear fractional differential equations. Advances in Dynamical Systems and Applications, 7 (1), 31-40.

[19] Khan, R. A. 2013. Three-point boundary value problems for higher order nonlinear fractional differential equations. Journal of applied mathematics \& informatics, 31 (12), 221-228.

[20] EL-Sayed, A.M.A., Bin-Taher, E.0. 2013. Positive solutions for a nonlocal multi-point boundary-value problem of fractional and second order. Electronic Journal of Differential Equations, 64 (3), 1-8.

[21] Shah, K., Khalil, H., Khan, R.A. 2015. Investigation of positive solution to a coupled system of impulsive boundary value problems for nonlinear fractional order differential equations. Chaos, Solitons \& Fractals, 77 (1), $240-246$.

[22] Samko, S.G., Kilbas, A.A., Marichev, O.I. 1993. Fractional Integrals and Derivatives: Theory and Applcations, Gordonand Breach, New York, NY, USA.

[23] Bashiri, T., Vaezpour, S.M., Park, C. 2016. A coupled fixed-point theorem and application to fractional hybrid differential problems. Fixed Point Theory and Applications, 23. 